\title{
Modelling spatio-temporal relevancy in urban context-aware pervasive systems using voronoi continuous range query and multi-interval algebra
}

\author{
Najmeh Neysani Samany ${ }^{\mathrm{a}}$, Mahmoud Reza Delavar ${ }^{\mathrm{b}, *}$, Nicholas Chrisman $^{\mathrm{c}}$ and \\ Mohammad Reza Malek ${ }^{\mathrm{d}}$ \\ ${ }^{a}$ Department of Surveying and Geomatics Engineering, College of Engineering, University of Tehran, \\ Tehran, Iran \\ ${ }^{\mathrm{b}}$ Center of Exellence in Geomatic Engineering in Disaster Management, Department of Serveying and \\ Geomatic Engineering, College of Engineering, University of Tehran, Tehran, Iran \\ ${ }^{\mathrm{c}}$ Department of Geomatic Science, Laval University, Québec, QC, Canada \\ ${ }^{\mathrm{d}}$ Department of GIS, Faculty of Geodesy and Geomatic Engineering, K.N. Toosi University of \\ Technology, Tehran, Iran
}

\begin{abstract}
Space and time are two dominant factors in context-aware pervasive systems which determine whether an entity is related to the moving user or not. This paper specifically addresses the use of spatio-temporal relations for detecting spatiotemporally relevant contexts to the user. The main contribution of this work is that the proposed model is sensitive to the velocity and direction of the user and applies customized Multi Interval Algebra (MIA) with Voronoi Continuous Range Query (VCRQ) to introduce spatio-temporally relevant contexts according to their arrangement in space. In this implementation the Spatio-Temporal Relevancy Model for Context-Aware Systems (STRMCAS) helps the tourist to find his/her preferred areas that are spatio-temporally relevant. The experimental results in a scenario of tourist navigation are evaluated with respect to the accuracy of the model, performance time and satisfaction of users in 30 iterations of the algorithm. The evaluation process demonstrated the efficiency of the model in real-world applications.
\end{abstract}

Keywords: Context-awareness, spatio-temporal relevancy, customization, multi interval algebra, range query, tourist

\section{Introduction}

Context appears as a fundamental key to enable systems to filter relevant information from what is available $[5,9,40]$, to choose relevant actions from a list of possibilities $[1,40]$, or to determine the optimal method of information delivery $[8,41]$. The major challenge of context-aware systems is the separation of the relevant from the irrelevant information $[12,40]$. This process requires finding an acceptable degree of information reduction, i.e., presenting as much information as needed and as little as required [40].

\footnotetext{
${ }^{*}$ Corresponding author: Mahmoud Reza Delavar, Center of Exellence in Geomatic Engineering in Disaster Management, Department of Serveying and Geomatic Engineering, College of Engineering, University of Tehran, Tehran, Iran. Tel.: +98 2161114257; +98 912; E-mail: mdelavar@ut.ac.ir.
} 
However, there are few reports concerning appropriate services to manage spatio-temporal relevancy parameters that determine whether a context is spatially related to the user or not.

Most of the current models for the spatio-temporal relevancy parameters in context-aware pervasive systems are based on the spatio-temporal relationships between the user and related objects. The main concentration of these models is on spatial relationships while in time dimension they usually applied temporal intervals $[12,29,40]$. Some studies have used the proximity relations between the user and the related contexts to model the spatial relevancy and utilised K-N neighbourhood or range queries [10, 29]. Such relations cover the inclusion of related contexts in a distinct area or range and the distance to other entities [10]. Stiller et al. [14] presented spatial user-item relations into recommender systems with distance functions and using weighting approaches. Holzmann and Ferscha [12] defined a ZoneOf-Influence (ZOI) for any entity with a specified distance and direction and used the RCC5 [3]. The position, direction and extension of both ZOI are also included in their model. The most important drawback of these systems is that they do not mention the characteristic of the user's movement in an urban network which typically follows a linear route with a specific direction and velocity $[30,32,33]$. However position, direction and velocity of a moving user have an important role in adaptation process. Moreover these approaches do not apply all of the topological relationships such as order relationships (e.g., behind or in- front- of), which could be useful in providing spatially relevant context-aware services. On the other hand in an urban network, the real distance between two objects is not the Euclidean distance but the actual network distance and applying K-N neighbourhood or range query without any modification will decrease the efficiency of such systems [15,18,26,27,31,35].

To model the spatio-temporal relevancy parameters in an urban context-aware system, which could cover the characteristics of the user's movement and utilise all of the spatial relationships (metric, directional and topologic) regarding to the time dimension, the approach proposed in this paper is organised into two main steps as follows:

(1) The quantitative representation of the moving user and his/her related contexts in space and time dimension with spatial and temporal intervals. The Spatial Interval of the user is Directed (DSI) and will be dynamically updated based on the position, velocity and direction of his/her movement. The Spatial Intervals (SIs) of the related contexts are assumed to be static and unchanging. The temporal intervals of the user (TIU) and related contexts (TIC) is not static and will be updated.

(2) Comprehensive representation of spatio-temporal relationships between the DSI and TI of the user and the SIs and TIs of the related urban contexts that can be modelled with the Voronoi Continuous Range Query (VCRQ) [25] and Multi Interval Algebra (MIA) [34].

The main contribution of this paper is the use of MIA for modeling spatio-temporal relevancy in an urban context-aware system. The selection of MIA in this research is considered for the following reasons: (1) when the user is moving in an urban network, he/she has a directional linear route. Therefore, the position of the moving user can be effectively modelled through a directed spatial interval $[33,34]$ that is adaptive to the user's behaviour (it can be extended or shortened according to the user's velocity). (2) In an urban area, one usually encounters solid objects; therefore, most of the related contexts of the user are considered with their external views which is sufficient to abstract the contexts and show them with spatial intervals. In the proposed approach "related contexts" refers to the entities that are preferred by the user. For example, the related contexts of a tourist are the attractive areas such as monuments, gardens and museums. (3) The relationships between the spatial and temporal intervals of the moving user and related contexts can manage spatio-temporal relationships in such a way that in space dimension all of the including topological, metric and directional relationships are covered. Figure 1 shows the importance of each spatial relationship. Most of the related contexts in an urban network have a valid 


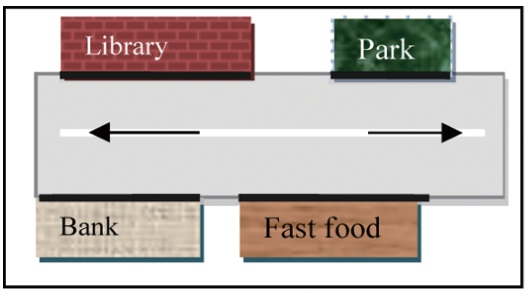

(a)

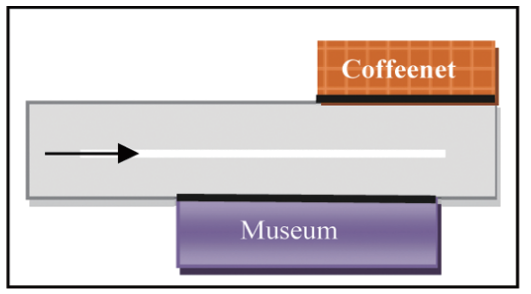

(b)

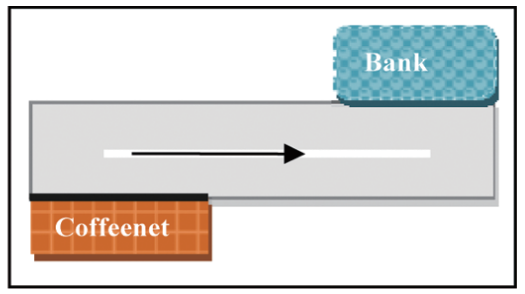

(c)

Fig. 1. The applications of MIA for specification of spatial relevant entities: a) the effect of the direction of the moving user, $b$ ) the effect of the distance between the moving user and related entities, c) the effect of topological relationships of the moving user and his/her related entities.

time to visit or to provide services, such as banks, shopping centers, libraries and etc. Therefore, it is necessary to specify temporal relationships between the moving user and his/her related contexts which lead to adapted services. (4) The algebra between spatial intervals and temporal intervals could introduce appropriate instructions (based on related contexts) to the user according to their arrangement in space. It should be noted that the algorithm is acted partially and followed up with the execution of a VCRQ [25]. Indeed, MIA assesses the spatio-temporal relationships between the user and the related contexts which are selected by the VCRQ.

Our approach is implemented in tow districts of Tehran, the capital of Iran, and we have focussed on an outdoor guided tour as an example. In this scenario, the user is a tourist who intends to visit some selected points of interest with a specified origin and destination. It is assumed that the tourist is equipped with a PDA or a laptop computer, and a GPS for positioning, and the route is constrained by a directed network. The origin and the destination of the user are recognised by the system or identified by the user. The proposed algorithm guides the user from the origin to destination. Guiding the user is adapted in modelling and representing procedures during the navigation task. The adaptation process is based on the time, position, direction and velocity of the user. The output of the system is the location and time information of hisher points of interest along the pre-defined route.

The evaluation process is based on three factors: the accuracy of the results, the time performance of the algorithm and the satisfaction of the users with the navigation process. The accuracy assessment of the model is based on the comparison of the expected and the actual spatio-temporally relevant objects detected by the model. The Chi-squared test is used to evaluate the goodness of fit. The experimental results show that the proposed approach can effectively model and accurately detect the spatio-temporally relevant contexts within a reasonable time frame. This approach also provides context-aware instructions for the user with a high percentage for user satisfaction.

The rest of this paper is structured as follows: Section 2 presents fundamental aspects concerning the spatio-temporal relevancy parameters and the principals of MIA theory with the approaches of customization. Then it describes the VCRQ and presents the research methodology and architectural design of the system. Section 3 presents a case study. The evaluation of the algorithm and the results obtained from the case study are explained in Section 4. Section 5 discusses the theoretical and practical issues of the proposed approach and attempts and gives a comprehensive comparison with related work. Finally, conclusions and directions of potential future research are considered in Section 6.

\section{Materials and methods}

In this section, the concept of spatio-temporal relevancy is described, followed by a description of the MIA theory and the approaches of customization. Then the principles of VCRQ are explained. Finally a 
description of the research methodology is introduced.

\subsection{Spatio-temporal relevancy in context-aware systems}

Saraceviec [41] offers a general definition of relevance derived from its general qualities: "Relevance involves an interactive, dynamic establishment of a relation by inference, with intentions towards a context. Relevance may be defined as a criterion reflecting the effectiveness of exchange of information between people (or between people and objects) in communication relation, all within a context". Three main relevancies in context-aware systems are identical relevancy, spatial relevancy and temporal relevancy $[10,42]$. Among these relevancies, the current position - 'the here' - is usually the centre of action, perception and attention. The identical information may be fully relevant at one position but irrelevant at another position $[6,8,17,37,42]$. Thus, the spatial relevancy is a parameter which has a fundamental role to provide context-aware services $[4,6]$. Similar to the 'here' in location humans are always at a certain point in time - the 'now'. This point of time is where one acts and also where one perceives the environment, analogous to the perception in space. The perception of contexts that are around is restricted to the point in time $[7,20]$. Therefore modeling of "spatio-temporal relevancy" is proved as an essential task in context-aware systems implementation.

\subsection{Multi intervals algebra}

Being similar to the well-known Interval Algebra developed for temporal intervals $[9,21]$ it seems useful to develop spatial interval algebra for modelling spatial relationships especially in an urban traffic network. When both temporal and spatial intervals for modelling spatial and temporal relationships between objects are applied, we encounter with Multi-Interval Algebra. The properties of spatial and temporal intervals and the interval algebra in time and space dimensions are described in Sub-sections 2.2.1 and 2.2.2.

\subsubsection{Temporal interval}

The Interval Algebra (IA) describes the possible relationships between convex intervals on a directed line. The default application of the Interval Algebra is temporal, so the considered line is usually regarded to be the timeline. In reality every events and objects start their life (are born) at $\mathrm{T}_{S}$, exists for a period of time (life span or duration) $\mathrm{T}_{D}$, and ceases to exist (die or finish) at $\mathrm{T}_{F}$. There are 13 temporal relations between two temporal intervals including before $<$, after $>$, meets $\mathrm{m}$, met-by mi, overlaps o, overlapped-by oi, equals $\equiv$, during d, include di, starts s, started-by si, finishes f, and finished by fi [9].

\subsubsection{Spatial interval}

There are several differences between spatial and temporal intervals which have to be considered when extending the Interval Algebra towards dealing with spatial applications [38]. The most important characteristic of spatial interval is its direction. A spatial interval can have the same or the opposite direction [23]. This leads to the definition of Directed Interval Algebra (DIA) with 26 base relations given in Renz [23], which result from refining each relation into two sub-relations specifying either the same or opposite direction of the involved intervals, and of all possible unions of the base relations.

\subsubsection{Spatio-temporal relationship in MIA}

Multi interval algebra defines spatio-temporal relationships between objects which could be represented with specified intervals in space and time dimensions. As we have 13 temporal relationships between temporal intervals and 26 spatial relationships between spatial intervals, there are $26 \times 13$ spatio-temporal relationships in MIA which can be represented by $M I A_{338}$. 


\subsection{Customizing $M I A_{338}$ to $M I A_{72}$}

Regarding to the characteristics of the moving user and related contexts in urban traffic networks, MIA could model spatio-temporal relevancy in an effective way [34]. The problem is that using all of the 13 relations in temporal domain and 26 relations in spatial domain or reasoning based on MIA $_{338}$ will reduce the speed of performance and it may decrease the efficiency of the context-aware system. Particularly when the user is moving with a specified velocity and he/she intends to make a decision due to the receiving messages of the system, the time of delivering appropriate instructions should be shortened as much as possible. Therefore it is necessary to reduce the existence spatio-temporal relationships in order to decrease computational complexity of the algorithm which leads to increase the time performance. Therefore the MIA 338 should be customized. There are two ways for customizing the Multi Interval Algebra [20,24].

(1) To use macro relations [28], i.e., unions of base relations. Indeed combining IA base relations and use these macro relations as base relations is the approach of customizing. For example, It is possible to combine $\{\mathrm{m}, \mathrm{o}\},\{\mathrm{mi}, \mathrm{oi}\},\{\mathrm{s}, \mathrm{d}, \mathrm{f}\},\{\mathrm{si}, \mathrm{di}, \mathrm{fi}\}$ and use them together with the relations $\{<\},\{>\}$, and $\{=\}$ as new base relations in space and time dimensions. This corresponds to the algebra $A_{7}$ defined in [28]. Regarding to the spatial and temporal intervals and their difference in MIA there are 7 relations in temporal dimension and 14 relations in spatial dimension. Therefore we have $14 \times 7$ or 98 spatio-temporal relationships with this type of customization or we have MIA98.

(2) To use only the relations which is needed, namely, the interval relations $<,>$, d, di, o, oi, $=$ and do not use m, mi, s, si, f, fi which correspond to intervals with common endpoints. This is similar to the algebra $\mathrm{A}_{6}$ defined in [28] with the exception of the identity relation. In this approach there are 6 relations in temporal dimension and 12 relations in spatial dimension. Therefore we have 72 spatio-temporal relationships with this type of customization or we have MIA T2 $_{2}$. This method of customization is applied in this paper.

\subsection{Voronio continuous range query}

This paper utilizes from VCRQ as $S-D$ continuous range search query which is defined as: "Retrieving all objects of interest on any point during the moving of the query point from the start point (S) to the destination (D) in the networks" [25]. In the continuous environment, when the query point is moving, it will cause a series of changes on the pattern of expected searching range in respect to the moving distance of the query point during the movement. Some objects could be moved out, others could be moved in. Therefor the time of updating should be specified [25]. The voronio continuous range query is carried out based on the proposed algorithm of Voronoi-based Range Search (VRS) which is defined in [25].

\subsection{The proposed spatio-temporal relevancy model}

This section presents our proposed approach for modeling spatio-temporal relevancy which is based on VCRQ and MIA. 


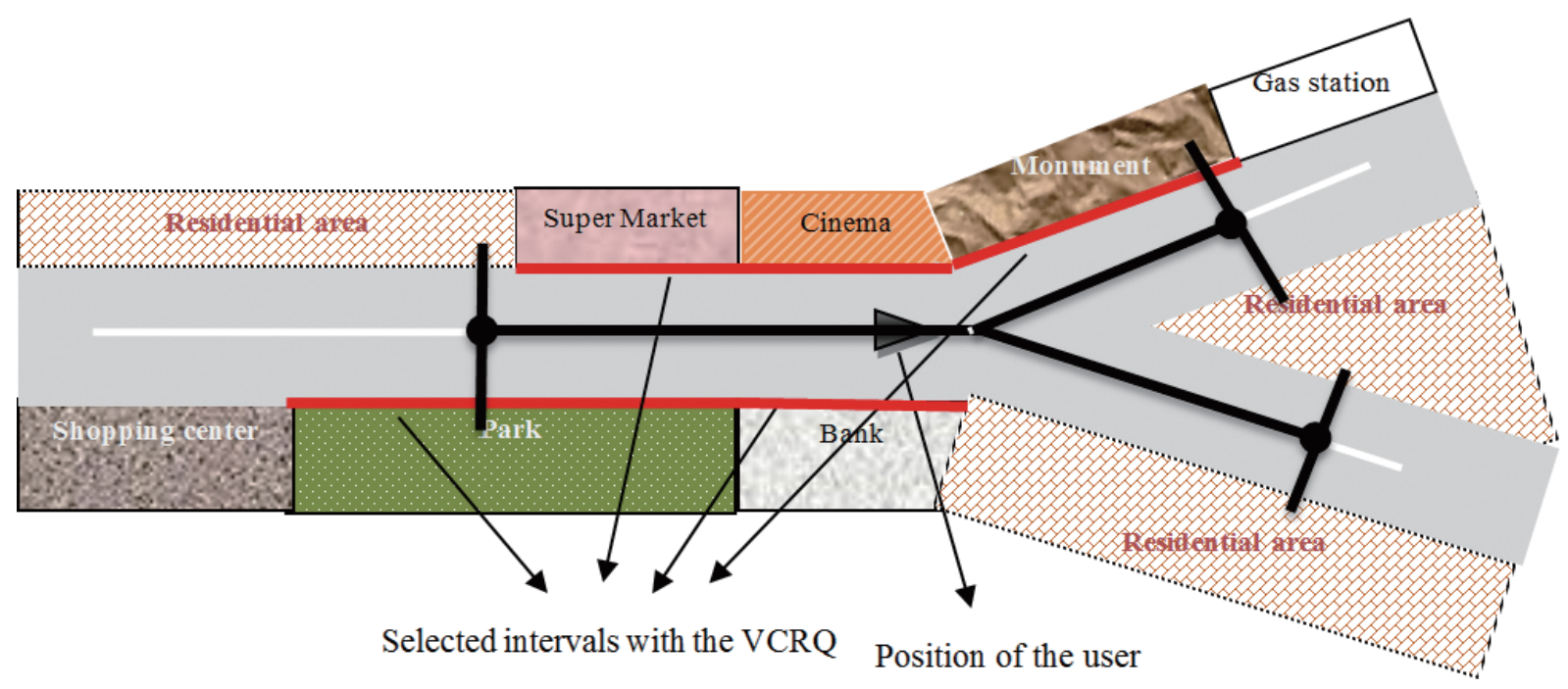

Fig. 2. The VCRQ for finding entities those are nearest to the user.

\subsubsection{The proposed algorithm}

The main steps of the proposed spatio-temporal relevancy model are summarised as follows:

1) The first step of the proposed algorithm is performing a voronio continuous range query [25], where the query point is the user's position. The results of this step are the related contexts which are near to the user based on the introduced searching range as illustrated in Algorithm 1. The searching range of VCRQ is changed based on the velocity of the user. It is computed as $\mathrm{e}_{V C R Q}=\mathrm{V} \subseteq \mathrm{t}$, where $\mathrm{V}$ is the velocity of the user $(\mathrm{m} / \mathrm{s})$ at the moment of updating and $\mathrm{t}(\mathrm{s})$ is the duration time of updating which is considered to be 6 seconds in this research (6 seconds are the minimum time required by the user to make each decision during the navigation task) (Fig. 2).

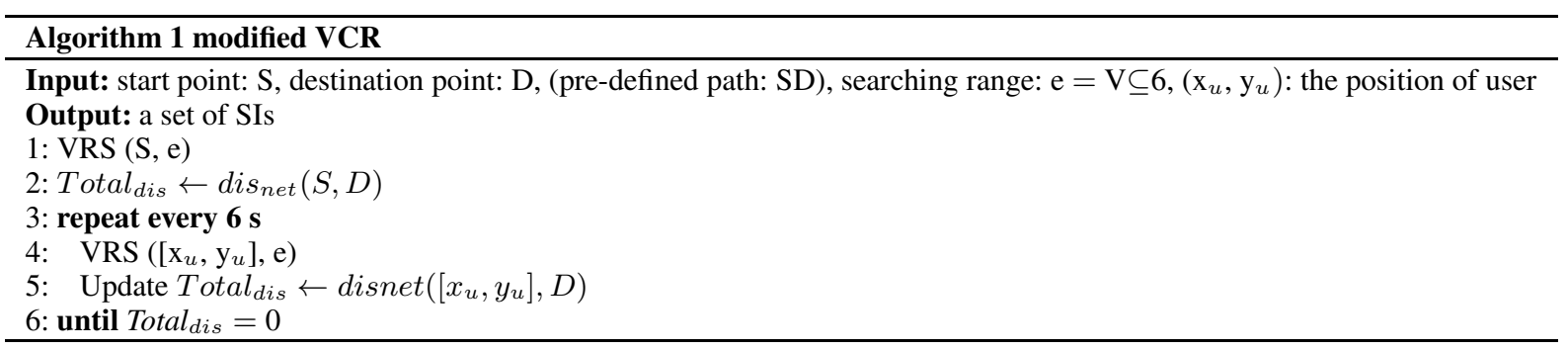

In Algorithm 1, VRS (Voronoi Range Search) [25] extracts some related contexts which are determined based on dynamic searching range (e) in every updating. Also is the network distance between two points [25].

2) The next step is the specification of the characteristics of the temporal interval and directed spatial interval of the user including its extension and direction which should be updated when the user moves. The positive and negative directions of the directed intervals are specified as shown in Fig. 3, in which the direction of the interval is determined based on its bearing of the interval (the computing approach of the bearing is presented in Appendix). If the positive and negative bearing of the intervals is between $0^{\circ}$ and $180^{\circ}\left(0^{\circ}=<\right.$ bearing $\left.<180^{\circ}\right)$, then the direction of the interval is 


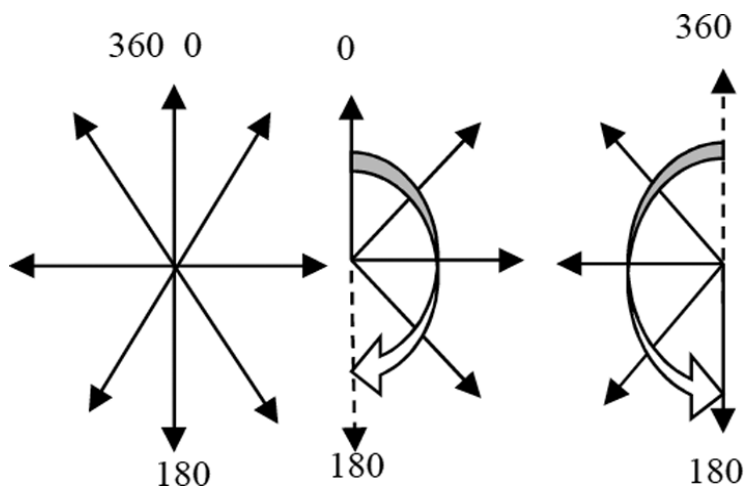

Fig. 3. Orientation relationships of the spatial directions.

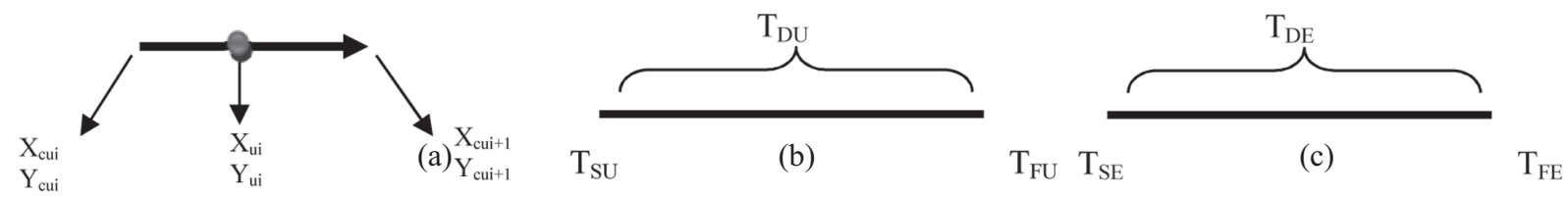

Fig. 4. A schematic view of (a) DSI (b) TIU (c) TIE.

positive $\left(\operatorname{dir}_{I}>0^{\circ}\right)$ and if the bearing of the intervals in between $180^{\circ}$ and $360^{\circ}\left(180^{\circ}=<\right.$ bearing $<360^{\circ}$ ), the direction of the interval is negative $\left(\operatorname{dir}_{I}<0^{\circ}\right)$. The extent of the spatial interval of the user is calculated using Eq. (1):

$$
\begin{aligned}
I_{u i}= & x_{c u i}=x_{u}-(V \times 6) \sin B_{i . i_{+1}} \\
& y_{c u i}=y_{u}-(V \times 6) \cos B_{i . i_{+1}} \\
& x_{c u i_{+1}}=x_{u}+(V \times 6) \sin B_{i . i_{+1}} \\
& y_{c u i_{+1}}=y_{u}+(V \times 6) \cos B_{i . i_{+1}}
\end{aligned}
$$

Where $\mathrm{I}_{u i}$ is the moving interval of the mobile user, $\mathrm{x}_{u}$ and $\mathrm{y}_{u}$ are the coordinates of the user's position, $\mathrm{A}_{i, i+1}$ is the bearing (B) of the direction ${ }_{i, i+1},\left(\mathrm{x}_{c u i}, \mathrm{y}_{c u i}\right)$ and $\left(\mathrm{x}_{c u i+1}, \mathrm{y}_{c u i+1}\right)$ are the coordinates of the start and end points of the directed interval respectively. As the velocity of the moving user in an urban traffic network varies, we consider $\mathrm{V}$ as the velocity of the user at the moment of an update and assume 6 seconds as the minimum time required by the user to make each decision during the navigation task. $\mathrm{V} \times \mathrm{t}$, which is equal to distance travelled during decision making process, is used the coefficient of the bearing. Figure 4(a) shows a schematic view of the directed intervals.

The extent of temporal interval of the user is specified as shown in Fig. 4(b) which is updated every 6 seconds. Where TSU is the start time of TIU which is equal to the current time of the moving user, TFU is finish time of TIU which is equal to the finish time of navigation which is introduced by the user at first and TDU is distance time between the TSU and TFU.

The appropriate spatio-temporal relationships between the DSI, SIs, TIU and TICs (which are determined by step1) are specified based on $\mathrm{MIA}_{72}$. The important aspect of the achieved instructions is that they will be sorted based on the distance to the user which is ranked after the execution of the VCRQ on step 1. It is fundamental that the user meets the nearer contexts before meeting the objects that are farther away. 
The procedure is repeated after every message update that is provided by the movement of the user. In this research we periodically update the position of the moving user every 6 seconds [16]. However the update of the position is done only when the user is on the move; when the user stops no update is performed. The direction of spatial interval of the user is updated based on the position update of the user.

Whenever the position of the user is updated, the spatial and temporal intervals of the user are constructed and the spatio-temporal relationships between the user and the nearest contexts (which are determined by the VCRQ) are evaluated. Based on the spatio-temporal relationships between the user and the detected contexts, the appropriate instruction is sent to the user. For example, if the spatial relationship is 'met from behind' and the temporal relation is 'contains' then the instruction is 'now you will arrive at place, you can visit it till 2:15 hours later", where "2:15 hours later" is the time duration that the related context will be closed to visit.

Temporal intervals of the related contexts are updated based on their collected information including opening times and closing times in every days. If we consider temporal interval of the contexts as Fig. 4(c), then $\mathrm{T}_{S C}$ is the opening time of the related contexts from 0:0 to $24, \mathrm{~T}_{F C}$ is the first closing time of the related objects after $\mathrm{T}_{S C}$ from 0:0 to 24 and $\mathrm{T}_{D C}$ is the distance time between the $\mathrm{T}_{S C}$ and $\mathrm{T}_{F C}$. It should be noted that this duration should be continuous. To achieve this goal, after any closing with opening of the related contexts, the temporal interval is updated. The pseudo-code for the proposed spatio-temporal relevancy is given in Algorithm 2.

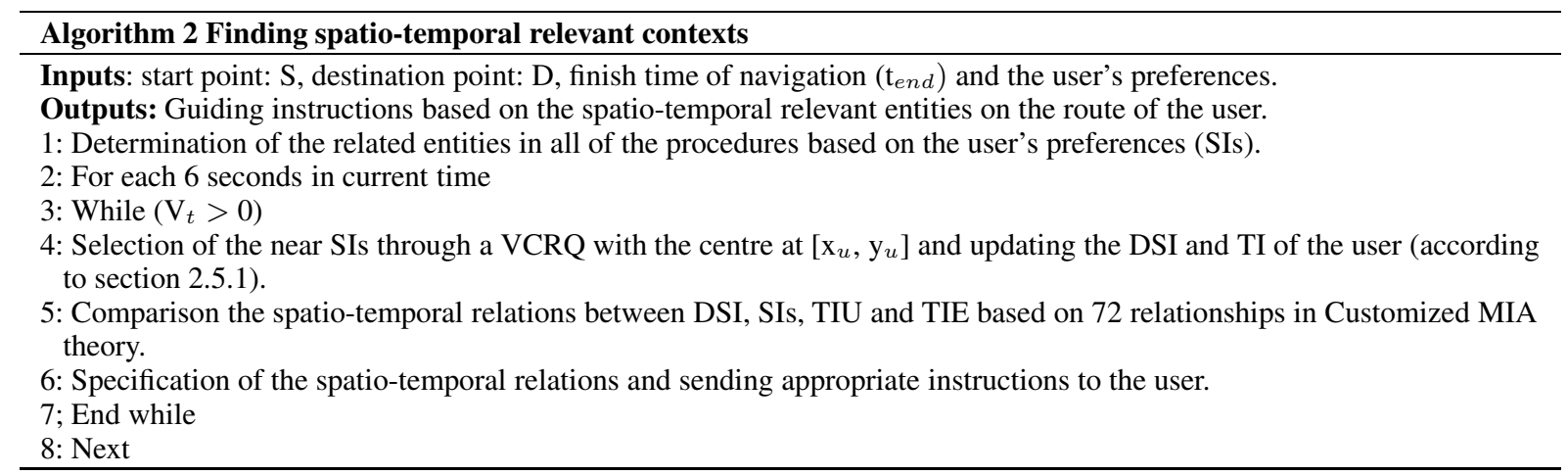

\subsubsection{Architectural design of the system}

The proposed spatio-temporal relevancy model is independent of the software and the programming language. In this study the user is a tourist with a specified scenario and the software is designed accordingly.

\subsubsection{System scenario}

In this research, the user is a tourist who is supposed to be guided from a hotel, which is his/her origin; if the location of the origin is not known, the user should introduce his/her current location to the system, also he/she should specify the finish time of the navigation process according to his/her schedule. After inputting the information about the origin, the user selects his/her point of interest (destination) based on his/her preferences (the characteristics of the places are introduced textually).

The system determines the optimum route between the origin and the destination. While moving, the tourist can be provided with location and time information of the other points of interest that are along 


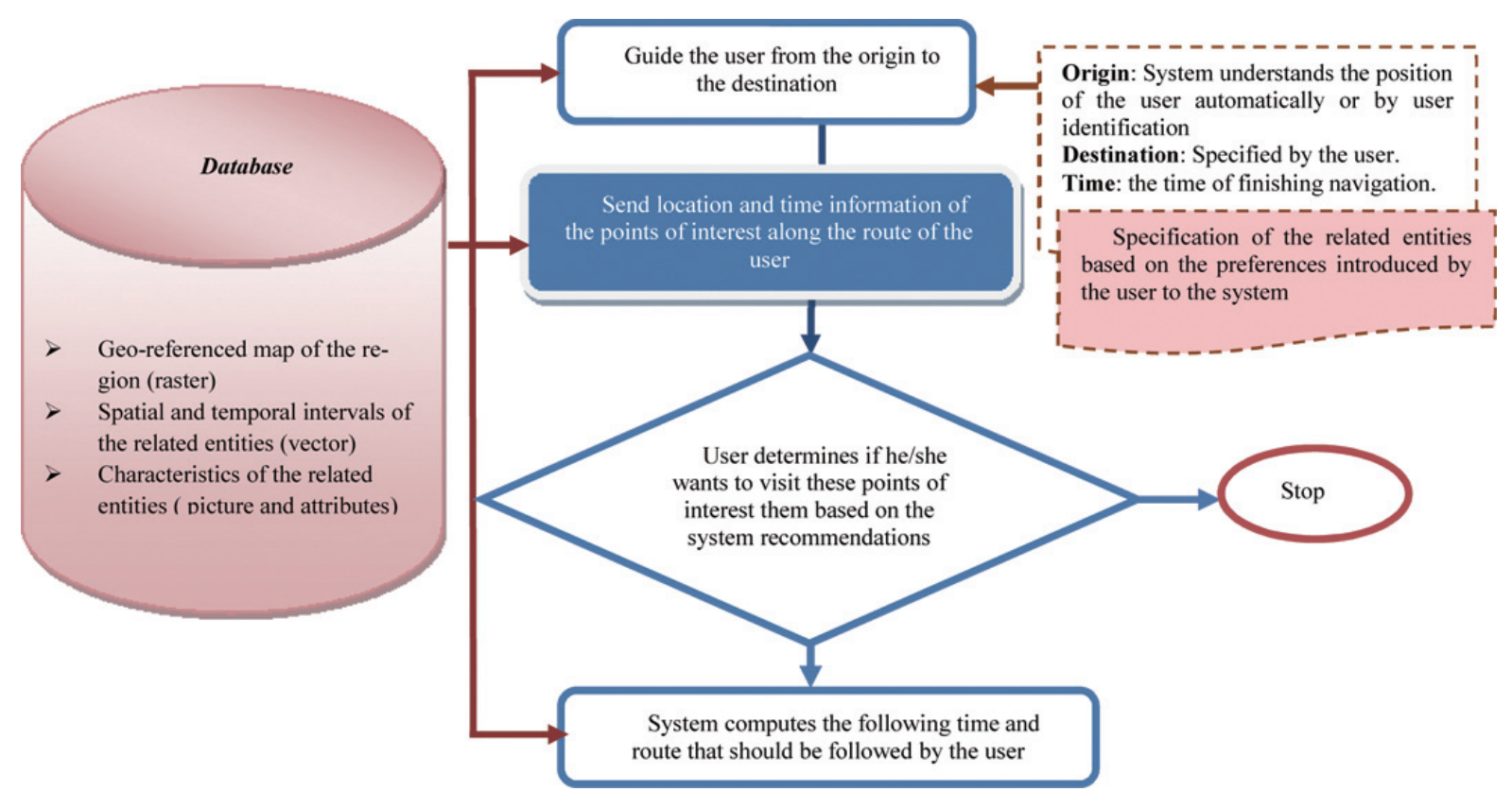

Fig. 5. A schematic view of the tourist scenario.

the route of the user (including the characteristics of the places shown on the user screen). Keeping track of the locations of services along the route, the tourist can obtain get an overview of the place where the points of interest are located. Moreover the user-adaptive system can direct the tourist when he/she is near such a spot. Figure 5 depicts a schematic view of the tourist scenario.

\subsubsection{Hardware architecture of the system}

The hardware architecture of the system consists of three main units for the correct display of contextaware services (Fig. 6).

\section{Implementation and case study}

The proposed spatio-temporal relevancy model for an urban context-aware system is implemented in the windows application environment with the Vb.Net. programming language, using a four-stage configuration wizard (Fig. 7(a),(b), (c) and (d)), and is delivered as the STRMCA software in the form of an exe. (or set-up). The set-up file has a feature for downloading the data of the region of interest. The applied spatial data are in vector format; however, we also considered a raster map as a background. The required data can be classified into static and dynamic data which are described as follows:

\subsection{Spatial data}

Static data are the information whose spatial characteristics are fixed during the navigation process. We have a raster map of the region as the background and a generalised georeferenced vector map with the following layers (all data used are at the scale of 1:2000):

- Graph of road network: It consists of the centreline of urban roads directed based on the urban rules (such as one-side and two-side). 


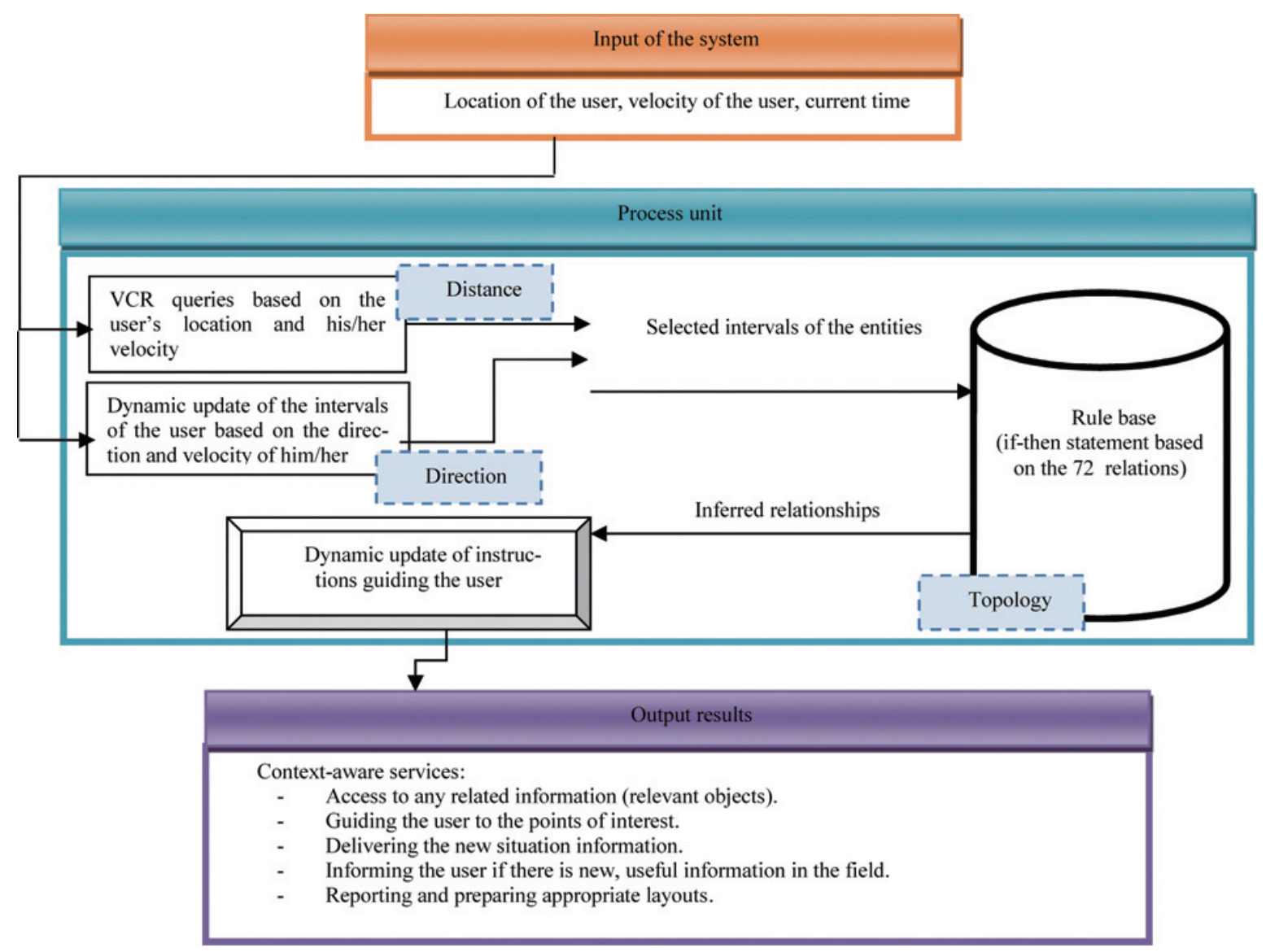

Fig. 6. Overview of the system architecture.

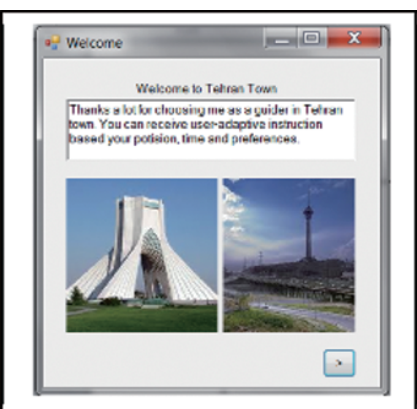

(a)

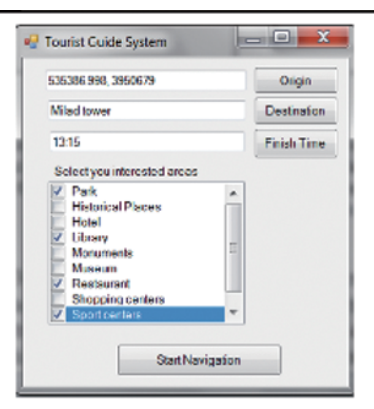

(b)

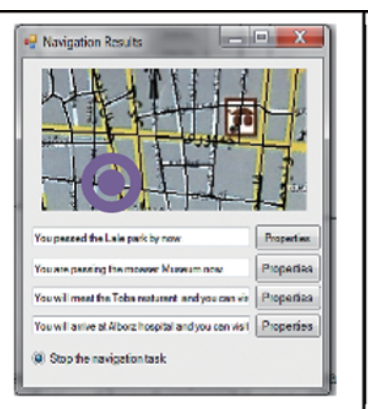

(c)

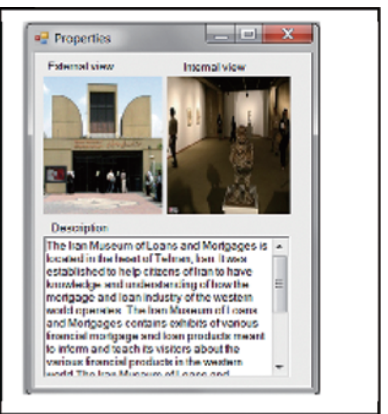

(d)

Fig. 7. The configuration wizard of the implemented system: a) welcome page, b) introduction of the origin and the destination to the user, specification of the preferences and the start of the navigation task, c) representation of context-aware instructions to the user and highlights of the spatially relevant contexts, d) illustration of pictures and characteristics of the selected area.

- Spatial intervals of the tourist points of interest and urban facilities: Because the spatial relationships are calculated with respect to the spatial intervals of the related contexts, they should be stored in the system to have a context-aware instruction. The SIs are represented by straight lines that cover 
the external boundaries of artefacts. The related contexts of the tourist are categorised as: (1) the tourist's points of interest, which consist of restaurants, coffee shops, parks, green spaces, shopping centers, downtown centers, monuments, historical places, cultural heritage sites, universities, libraries, exhibitions, sport sites, museums and hotels. (2) The urban facilities that may be used by the user, such as petrol stations, hospitals, care centres, metro stations, bus stations and airports. The external boundary of the related contexts is digitised as a spatial interval, and the coordinates of the start and end points of the line are stored in the database of the system.

\subsection{Dynamic data (real-time data)}

Dynamic data are information that should be updated periodically with the user's movement. The computation process is carried out based on variations in the data. In this study the position, time and velocity of the user are the dynamic (real-time) data. Since almost all of the related contexts in an urban network have a valid time to visit. So the temporal intervals of them are updated accordingly.

\section{Experimental results and evaluation}

The spatio-temporal relevancy model is implemented in urban network of two districts of Tehran, the capital of Iran. Districts 3 and 6 cover some attractive areas for a tourist and are considered for our case study. To investigate how the model would perform in a real-world application, 30 different routes with different origins and destinations were selected. Each route was traversed by a visitor equipped with a laptop, a GPS and the software designed based on the proposed model. This paper evaluates the results of the experiments based on three parameters namely; the accuracy of the results [9], the performance of the model [12] and the satisfaction of the users [13].

\subsection{Accuracy of the results}

The metric employed for accuracy assessment of the proposed model is based on a comparison of the count of the number of contexts detected by the model and the related contexts that should be selected in the environment (control contexts) $[11,36]$. The Chi-squared $\left(\chi^{2}\right)$ statistic is selected for testing the proposed approach. Chi-squared test of goodness of fit establishes whether an observed frequency distribution differs from an expected distribution.

To test this parameter, 30 tourists traversed 30 different routes with different origins and destinations. In each route the related contexts selected by the tourist via the user's preference options are specified as control points regarding to their temporal constraints.

The system is run while the user moves, and the user is guided based on the spatio-temporally relevant contexts with ordered instructions. Then, the number of detected contexts in each route is compared with the control contexts. Figure 8 graphically depicts the difference between the two diagrams of the detected contexts and the control contexts.

Chi-squared goodness- of- fit test was used to compare the expected spatio-temporally relevant contexts; the results demonstrate the efficiency of the model based on the accuracy of the detected relevant contexts at $95 \%$ and $99 \%$ confidence levels. Table 1 shows the values of Chi-squared tests at $95 \%$ and $99 \%$ confidence levels. A comparison between the value of Chi-squared statistics shown in columns 3 and 4 of the Table 1 specifies the accuracy of the Chi-squared proposed algorithm. 
Table 1

Results of Chi-squared goodness of fit

\begin{tabular}{ccccc}
\hline Number of iterations & DF & $\chi 2$ & $95 \%$ Confidence level & 99\% Confidence level \\
\hline 30 & 29 & 2.423 & 17.708 & 14.256 \\
\hline
\end{tabular}

Table 2

Time in (s) for selecting related entities based on the distance parameter with the VCRQ

\begin{tabular}{lccc}
\hline The number of related entities & $1-5$ & $5-10$ & $10-15$ \\
\hline Time (second) & $0.03-0.30$ & $0.3-0.48$ & $0.48-0.59$ \\
\hline
\end{tabular}

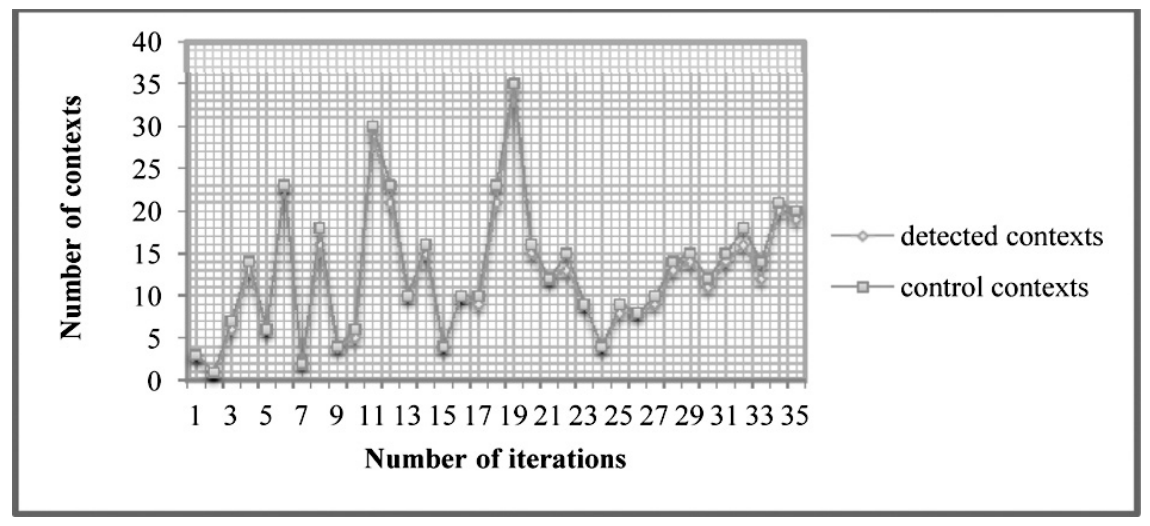

Fig. 8. Comparison between the control entities and detected the entities.

The results of the comparison between the values shown in Table 1 indicate that in 30 iterations of the algorithm in 30 different routes, the p-value of the Chi-squared test is significant. In other words, the value of $\chi 2$ which is equal to 2.423, is smaller than of errors at a 95\% confidence level (17.708) and 99\% confidence level (14.256) with the Degree of Freedom (DF) equal to 29. Thus, the statistics demonstrate that the proposed approach can accurately model spatio-temporal relevancy in a context-aware system.

It should be noted that the introduced model is utilizing from VCRQ despite of Dynamic Range Neighbour Query (DRNQ) which was applied in our previous work [33]. The achieved results based on the comparison between the current approach and previous research demonstrated that using VCRQ increases the efficiency of the system by decreasing the number of false or undetected contexts (the details of this comparison are not mentioned in this paper).

\subsection{Performance of the model}

In this section, the results of tests that have been performed to show the run-time efficiency of the algorithm are presented. Three performance tests were conducted, for which a Windows 7 Ultimate system (Intel ${ }^{\circledR}$ Atom (TM) CPU N270 and 2GB RAM) was used. The first set of results shows how much time is needed for updating a VCRQ based on the user's position (Table 2). The second evaluation reveals the measured time that is required for providing context-aware instructions, which consists of updating the directed spatial interval and temporal intervals based on the time, position, direction and velocity of the moving user (Table 3). The final performance evaluation parameter is the required time for implementing the proposed model in any updating procedure including (1) running a VCRQ, (2) updating the DSI and TI of the user, specifying of the type of spatio-temporal relationships between the 
Table 3

Time in (s) for updating DSI and TI of the user and introducing spatio-temporal relevant entities to the user in any update

\begin{tabular}{lccc}
\hline The number of spatio-temporal relevant entities & $1-5$ & $5-10$ & $10-15$ \\
\hline Time (second) & $0.03-0.18$ & $0.18-0.27$ & $0.27-0.34$ \\
\hline \multicolumn{5}{c}{ Table 4} \\
Time in (s) for introducing spatio-temporal relevant entities to the moving user in every update \\
\hline Total time (second) & $1-5$ instruction/s & $5-10$ instructions & $10-15$ instructions \\
\hline Time & $0.06-0.58$ & $0.58-1.03$ & $1.03-1.25$ \\
\hline
\end{tabular}

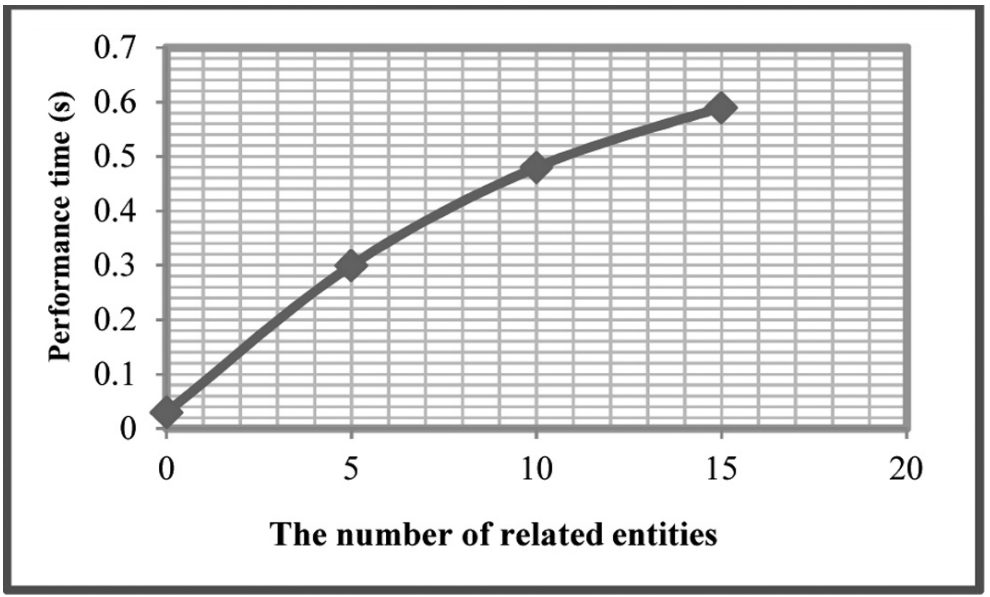

Fig. 9. Time taken for the VCRQ.

DSI, SIs, TIU and TIC and giving appropriate instructions to the user (Table 4). It should be noted that the updating of TI of the related contexts is performed by the system software whenever it is needed.

The archived results reveal that the processing time depends on the number of related contexts (SIs) around the user; however, similar times have been measured for a smaller number of SIs with a correspondingly large number of related contexts.

Up to 15 SIs in the study area around the user area have been considered (based on the constraints in an urban network, 15 is the maximum number of SIs that are detected in the updating algorithm). The measured time for building a VCRQ, updating the DSI and TI of the user and providing context-aware instructions and the total time for the whole procedure are shown in Figs 9, 10 and 11. The aim of the performance evaluation was to investigate how much time is needed for a particular test system for performing the respective tasks with variable numbers of SIs. The results achieved demonstrate that the total computation time of STRMCA performance in the study area is less than $1 \mathrm{~s}$ and users have $5 \mathrm{~s}$ for decision making.

\subsection{Satisfaction of the users}

The final parameter for the assessment of the system model is the satisfaction of the user with the system assistance procedure. The user's satisfaction with the services provided is a key issue in modeling context-aware systems $[11,13]$. It is obviously crucial for adoption and acceptance of such technologies [12,39]. This study considered seven elements for estimating user satisfaction from the model and 


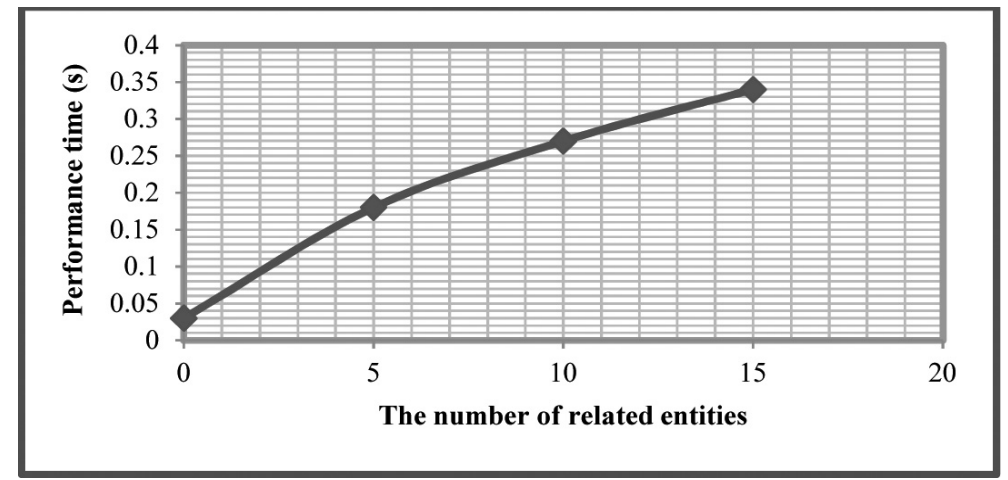

Fig. 10. Time taken for updating, the DSI and TIU and providing user-adaptive instructions according to the number of SIs.

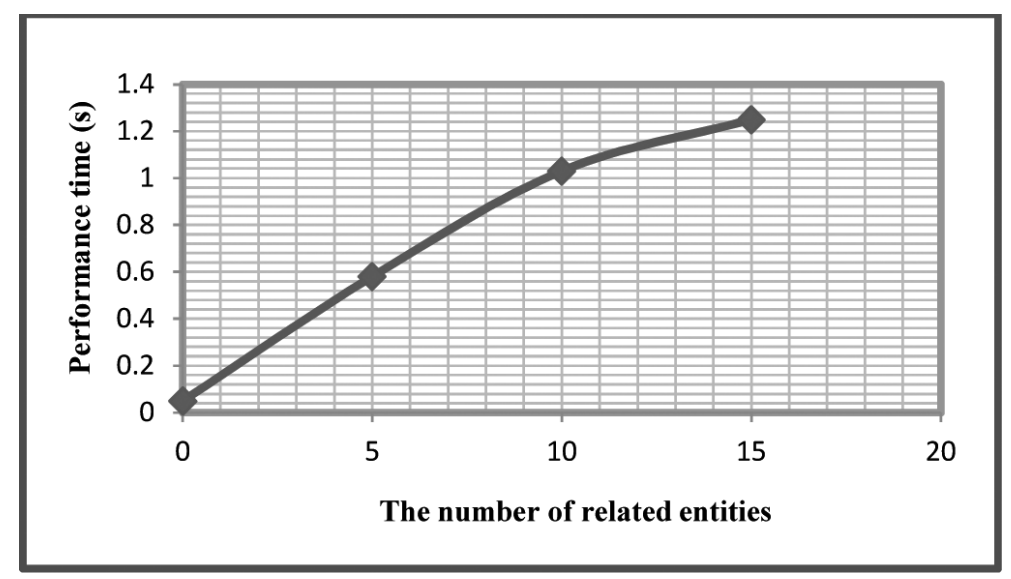

Fig. 11. Time performance of the proposed algorithm.

context-aware services, namely: (1) the usefulness of the prediction in the user's decision making process for choosing his/her related contexts $[2,13],(2)$ the usefulness of the presentation of contexts that have spatial relationships behind the user and in the opposite direction, (3) the effect of the contexts introduction based on the velocity (this factor is derived from the "adapt to varying situations of the user" parameter pointed by [12], (4) the order of the contexts presentation and (5) the responding time of the algorithm to the user [3,12], (6) number of navigation steps [13], and (7) task success [13].

To achieve this goal, a questionnaire was designed and given to each visitor. All 30 tourists completed the questionnaire at the end of the process and gave a yes or no score for each parameter. For every parameter, the number of visitors who agreed or disagreed with the satisfaction factor was obtained. Table 5 shows the percentages of the visitors who agreed and those who disagreed for each parameter.

The statistical analysis of the results obtained from questionnaires completed by the visitors demonstrated that on average more than $90 \%$ of the visitors agreed with the method implemented in STRMCAS for presenting approach of navigation instructions (Fig. 12). The results shown in Table 6 indicate a high level of user satisfaction with the proposed model. 
Table 5

The percentage of the satisfaction of the users with each parameter

\begin{tabular}{clcc}
\hline No. & Satisfaction parameter & \multicolumn{2}{c}{ Score } \\
\cline { 3 - 4 } & & Yes & No \\
\hline 1 & Usefulness of the related entities prediction & $92 \%$ & $8 \%$ \\
2 & Usefulness of the presentation of entities that have spatial relationships & $84 \%$ & $16 \%$ \\
& behind the user and in the opposite direction & & \\
3 & Effect of the entities introduction based on the velocity & $88 \%$ & $12 \%$ \\
4 & The order of the entities presentation & $92 \%$ & $8 \%$ \\
5 & Response time & $85 \%$ & $15 \%$ \\
6 & Number of navigation steps & $88 \%$ & $12 \%$ \\
7 & Task success & $95 \%$ & $5 \%$ \\
\hline
\end{tabular}

Table 6

Classification of the level of user satisfaction level of the users

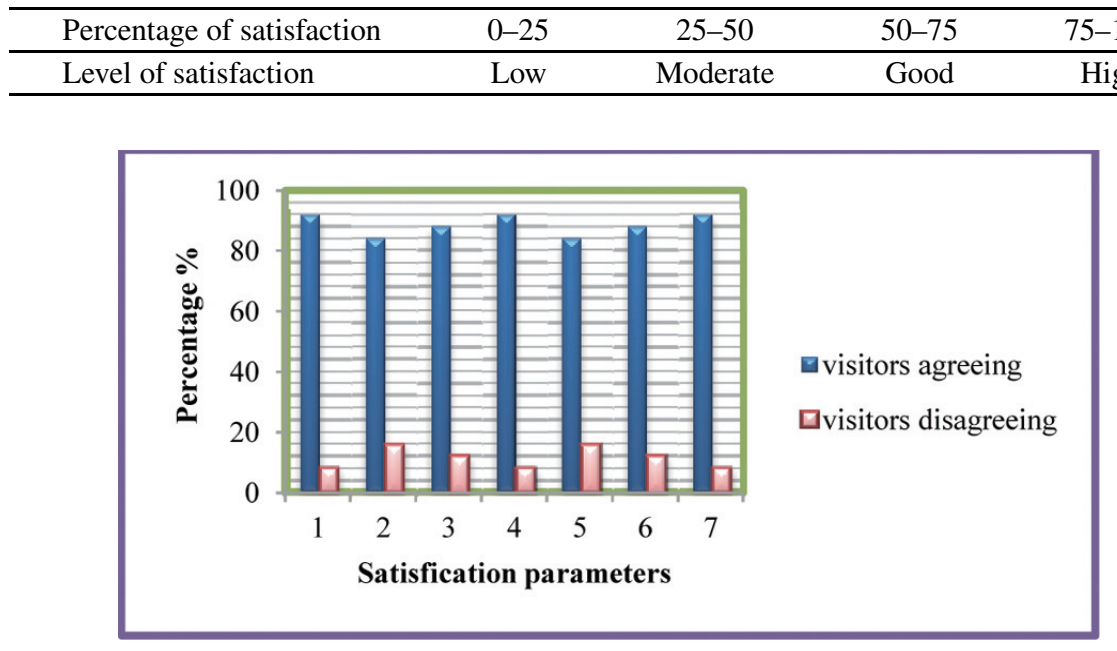

Fig. 12. The comparison of the rate of satisfaction.

\section{Discussion}

This study proposes and verifies a new approach to model the spatio-temporal relevancy parameter in context-aware systems constrained by directed urban traffic network. The proposed model has some specific characteristics that make this model distinctly different from current models. The first key feature of this model is the consideration of the influence of the moving user with a directed spatial interval despite of considering it as a point or a region. A directed spatial interval can model the movement characteristics of the user in an urban network effectively because it includes the direction of the user, which is needed for deciding about continuing/returning/stopping the route. Such a model can also characterise the velocity of the user's movement by decreasing and increasing the size of the user's directed spatial interval; thus, whenever the velocity increases, the DSI is extended, and when the velocity decreases, the DSI is shortened. Therefore, in the former case, the more SIs are considered to have spatial relationships with the DSI and the user have sufficient time to make a decision about whether to visit the related contexts. In the latter case, because of the low velocity of the user, the fewer contexts are found, and the user can decide to visit a place for a longer time (Fig. 13).

To evaluate the model in real-world applications, three metrics were considered (Sections 4.1, 4.2 and 
Table 7

The comparison between a few related projects

\begin{tabular}{|c|c|c|c|c|}
\hline Related work & SAPM (2008) & Relate Project (2009) & ZOI (2010) & Proposed model \\
\hline \multicolumn{5}{|l|}{ Parameters } \\
\hline Distance relations & $\sqrt{ }$ & $\sqrt{ }$ & $\sqrt{ }$ & $\sqrt{ }$ \\
\hline Topological relations & $\begin{array}{l}\text { 9-Interaction } \\
\text { Model }\end{array}$ & $\begin{array}{l}\text { left-of, right-of, } \\
\text { approaching, moving away }\end{array}$ & RCC5 & $\begin{array}{l}\text { RCC8 and ordered } \\
\text { through MIA }\end{array}$ \\
\hline Directional relations & $\sqrt{ }$ & $\sqrt{ }$ & $\sqrt{ }$ & $\sqrt{ }$ \\
\hline Velocity of the user & $x$ & $x$ & $x$ & $\sqrt{ }$ \\
\hline $\begin{array}{l}\text { Consideration of the } \\
\text { influence domain }\end{array}$ & $x$ & $\sqrt{ }$ & $\sqrt{ }$ & $\sqrt{ }$ \\
\hline $\begin{array}{l}\text { Arrangement of the contexts } \\
\text { in spatial dimensions }\end{array}$ & $x$ & $\sqrt{ }$ & $\sqrt{ }$ & $\sqrt{ }$ \\
\hline Temporal relations & $\begin{array}{l}\text { Time is considered } \\
\text { as an attribute }\end{array}$ & $\begin{array}{l}\text { Time is considered } \\
\text { as an attribute }\end{array}$ & Interval Algebra & Interval Algebra \\
\hline
\end{tabular}

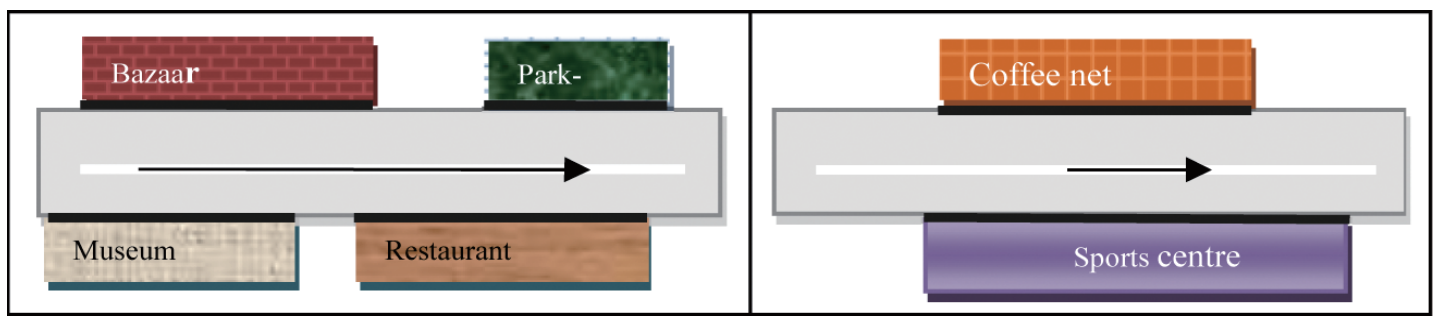

(a)

(b)

Fig. 13. Dependency of the size of the DSI to the velocity of the user: a) at a higher speed, b) at a lower speed.

4.3). The results of the evaluation demonstrated that the algorithm is able to accurately detect the spatiotemporally relevant contexts of a moving user in an appropriate run-time and with a high level of user satisfaction with the system's performance. Also the comparison results between the current approach and previous research [33] demonstrated that using VCRQ increases the efficiency of the system by decreasing the number of false or undetected contexts. Furthermore the satisfaction of the users by using VCRQ in the first step of the proposed algorithm leas to the $2 \%$ increase rather than when we used DRNQ [33].

Another parameter which is important in context-aware system is usability of the model [13] STRMCAS have 4 main components for modeling spatio-temporal relevancy (DSI, SI, TIU and TIC) and it is possible for every navigation applications to adapt with defining them. Indeed only the related contexts are changed based on the user preferences and goals.

While the advantages of the proposed spatio-temporal relevancy model are clearly evident, there are also some limitations to this research which should be addressed in future studies. Although the model handles spatio-temporal relevancy in urban traffic networks, it may not be suitable for a pedestrian user and may be not applicable in indoor spaces that do not have directed networks, such as a museum.

\subsection{Comparison with related works}

This section gives an overview of the related works that address the use of qualitative and quantitative spatio-temporal relationships to select relevant objects. The comparison is performed based on the characteristics of the STRMCAS with regard to other closely related studies and projects. One of 
these studies is the research of Holzmann and Ferscha [12], who defined ZOI for contexts and specified the direction, distance spatial relationships between related sensors. Gellersen et al. [19] expressed the quantitative relationships by the distance between devices and the orientation angle, and the qualitative relationships by the spatial arrangement of one device with respect to another. The Spatial Audit Policy Model (SAPM) introduced the concept of the spatial audit rule and supported the homogeneous representation of all spatial aspects involving objects and adapted information such as the user's position, with a 9-intersection topological approach [43]. The comparison of these projects with respect to the different aspects concerning spatial relationships and adaptation parameters is given in Table 7.

As seen from Table 7, using spatio-temporal relationships is a common technique for spatio-temporal relevancy modelling but in no case are all of the spatial relations such as metric, directional and topological (with all mutually ordered relationships) considered. In adapting the model to the user's movement characteristics there is no method that incorporates the velocity of the user in providing spatially relevant objects to the user. The consideration of the influence domain is an essential assumption in the recent studies, but the innovation of this paper is the definition of linear spatial intervals for a user and his/her related contexts. Also it can be deduced that presenting spatial related contexts in a real order is needed for user convenient which is considered in the proposed approach effectively.

\section{Conclusions and future directions}

The main contribution of this paper is the specification of a model for spatio-temporal relevancy, which is adapted to the characteristics of moving user in an urban traffic network, and its implementation in a tourist guide system. The model enables context-aware services to be managed without the user's prior knowledge of the area. Adaptation of the application to the user is based on the Voronoi Continues Range Query and Multi Interval Algebra. With customizing the spatio-temporal relationships of MIA, 72 spatial relationships between intervals of the user and related contexts are specified to detect the spatio-temporal relevant contexts.

In this research the tourist guide is equipped with a PDA or Laptop system and a tool for positioning system like GPS. The tourist could execute this program in his/her device and receive the expected context-aware service conveniently. The experimental results show that the proposed approach could detect spatio-temporal relevant contexts at the right position at the right time with a high level of satisfaction. The results of accuracy with Chi-square fitness-of-use and time performance demonstrated the implemented model. Also the evaluation of filled questionnaire form of user indicated that the proposed approach could satisfy the user in providing and introducing context-aware services.

\section{Appendix}

In this section we explain an approach of bearing computation for a directed line. Consider a line $\left(\mathrm{L}_{A B}\right)$ with the origin of $\mathrm{A}$ and destination of $\mathrm{B}$. Indeed the direction of $\mathrm{L}_{A B}$ is from $\mathrm{A}$ to $\mathrm{B}$. Bearing of $\mathrm{L}_{A B}\left(\mathrm{~B}_{A B}\right)$ is a clockwise angle from the magnetic north to the directed line (Fig. a) which is computed as Eq. (a), Eq. (b), Eqs. (c) and (d) [44]:

$$
\begin{aligned}
& \text { If } \Delta x>0 \text { and } \Delta y>0 \\
& \text { then } \mathrm{B}_{A B}=\arctan (\Delta \mathrm{x} / \Delta \mathrm{y}) \\
& \text { If } \Delta x>0 \text { and } \Delta y<0 \\
& \text { then } \mathrm{B}_{\mathrm{AB}}=180^{\circ}-|\arctan (\Delta \mathrm{x} / \Delta \mathrm{y})|
\end{aligned}
$$




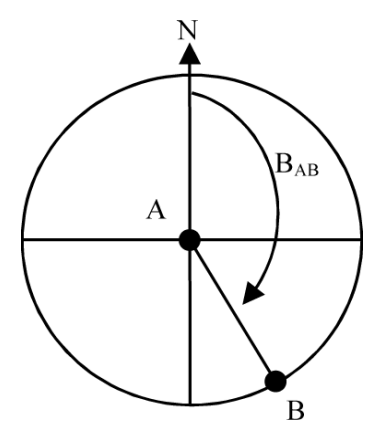

Fig. a. Bearing of the direction AB.

If $\Delta x<0$ and $\Delta y>0$

then $\mathrm{B}_{\mathrm{AB}}=180^{\circ}+|\arctan (\Delta \mathrm{x} / \Delta \mathrm{y})|$

If $\Delta x<0$ and $\Delta y<0$

then $\mathrm{B}_{\mathrm{AB}}=360^{\circ}-|\arctan (\Delta \mathrm{x} / \Delta \mathrm{y})|$

where $\Delta x=x_{B}-x_{A}$ and $\Delta y=y_{A}-y_{B}$

\section{References}

[1] A. Coronato and G. Pietro, Formal specification and verification of ubiquitous and pervasive system, ACM Transactions on Autonomous and Adaptive Systems 6(1) (2011), 9.

[2] A.D. Kshemkalyani, Immediate detection of predicates in pervasive environments, J Parallel and Distrib Comput 72 (2012), 219-230.

[3] A.G. Cohn, B. Bennett, J. Gooday and N.M. Gotts, Representing and reasoning with qualitative spatial relations about regions, in: Spatial and Temporal Reasoning, Kluwer Academic Publishers, 1997, pp. 97-134.

[4] A. Jimenez-Molina and I.Y. Ko, Spontaneous task composition in urban computing environments based on social, spatial and temporal aspects, J Eng Applications of Artificial Intelligence 24 (2011), 1446-1460.

[5] A.K. Dey, Understanding and using context, Personal and Ubiquitous Computing 5 (2001), 4-7.

[6] A. Schmidt, Ubiquitous computing - computing in context, PhD Thesis, Lancaster University, 2002.

[7] A.U. Frank, Tiers of ontology and consistency constraints in geographical information systems, International Journal of Geographical Information Science 15(7) (2001), 667-678.

[8] B.N. Schilit, N. Adams and R. Want, Context-aware computing applications. In: proceedings workshop on mobile computing systems and applications, Santa Cruz, California, USA Dec. 8-9, 1994, pp. 85-90.

[9] B.Y. Lim and A.K. Dey, Assessing demand for intelligibility in context-aware applications, UbiComp 2009, Sep 30-Oct 3, Orlando, Florida, USA, 2009, pp. 195-204.

[10] C. Becker and D. Nicklas, Where do spatial context-models end and where do ontologies start? A proposal of a combined approach, In: Proceedings of First International Workshop on Advanced Context Modelling, Reasoning and Management in conjunction with UbiComp 2004, 2004, pp. 48-53.

[11] C. Gena, Methods and techniques for the evaluation of user-adaptive systems, The Knowledge Engineering Review 20(1) (2005), 1-37.

[12] C. Holzmann and A. Ferscha, A framework for utilizing qualitative spatial relations between networked embedded systems, Journal of Pervasive and Mobile Computing 6 (2010), 362-381.

[13] C. Mulwa, S. Lawless, M. Sharpm and V. Wade, The evaluation of adaptive and personalised information retrieval systems: A review', Int J Knowledge and Web Intelligence 2(2/3) (2011), 138-156.

[14] C. Stiller, F. Ro and Ch. Ament, Integration of Spatial User-Item Relations into Recommender Systems, International Journal for Infonomics (IJI), Infonomics Society 3(1) (2011), 190-196.

[15] D. Taniar, M. Safar, Q.T. Tran, W. Rahayu and J.H. Park, Spatial network RNN queries in GIS, The Computer Journal 54(4) (2011), 617-627.

[16] G. Iwerk, Maintenance of spatial queries on continuously moving points. PhD Thesis, University of Maryland at College Park College Park, MD, USA, 2004. 
[17] G. Tychogiorgos and Ch. Bisdikian, Selecting relevant sensor providers for meeting "your" quality information needs,. Proc. IEEE Conference on Mobile Data Management (MDM), 2011, Lulea, Sweden.

[18] G. Zhao, K. Xuan, D. Taniar and B. Srinivasan, Incremental k-nearest-neighbor search on road networks, Journal of Interconnection Networks 9(4) (2008), 455-470.

[19] H. Gellersen, C. Fischer, D. Guinard, R. Gostner, G. Kortuem, C. Kray, E. Rukzio and S. Streng, Supporting device discovery and spontaneous interaction with spatial references, Journal of Personal and Ubiquitous Computing 13(4) (2009), 255-264.

[20] I. Afyouni, C. Ray and Ch. Claramunt, Spatial models for indoor and context-aware navigation systems: A survey, $J$ Spatial Info Sci 4(1) (2011), 85-123.

[21] J.F. Allen, Maintaining Knowledge about temporal intervals. Comm, ACM 26(11) (1983), 832-843.

[22] J. Hong, E.-H. Suh, J. Kiim and S. Kim, Context-aware system for proactive personalized service based on context history, Journal of Expert Syst. with Applications 36 (2009), 7448-7457.

[23] J. Renz, A spatial odyssey of the interval algebra: Directed intervals, in: Proceeding of the 17th Znt 'I Joint Conference on Artificial Intelligence, B. Nebel, ed., Morgan Kaufmann Publishers Inc. San Francisco, CA, USA, August, 2001, pp. 51-56.

[24] J. Renz and F. Schmid, Customizing qualitative spatial and temporal calculi, M.A. Orgun and J. Thornton, eds, AI 2007, LNAI 4830, pp. 293-304, Springer-Verlag Berlin Heidelberg.

[25] K. Xuan, K. Xuan, G. Zhao, D. Taniar, W. Rahayu, M. Safar and B. Srinivasana, Voronoi-based range and continuous range query processing in mobile databases, Journal of Computer and System Sciences 77 (2011), 637-651.

[26] K. Xuan, G. Zhao, D. Taniar and B. Srinivasan, Continuous Range Search Query Processing in Mobile Navigation, Proceedings of the 14th International Conference on Parallel and Distributed Systems (ICPADS 2008), IEEE, 2008, pp. 361-368.

[27] K. Xuan, G. Zhao, D. Taniar, M. Safar and B. Srinivasan, Constrained range search query processing on road networks, Concurrency and Computation, Practice and Experience 23(5) (2011), 491-504.

[28] M.C. Golumbic and R. Shamir, Complexity and algorithms for reasoning about time: A graph theoretic approach', $J$ of the ACM 40(5) (1993), 1128-1133.

[29] M. Grossmann, M. Bauer, N. Honle, U. Kappeler, D. Nicklas and T. Schwarz, Efficiently managing context information for large-scale scenarios, in: Proceedings of Pervasive Computing and Communications, IEEE Computer Society, 2005, pp. 331-340.

[30] M. Safar, D. Ebrahimi and D. Taniar, Voronoi-based reverse nearest neighbor query processing on spatial networks, Multimedia Systems 15(5) (2009), 295-308.

[31] M. Safar, D. El-Amin and D. Taniar, Optimized skyline queries on road networks using nearest neighbors, Personal and Ubiquitous Computing 15(8) (2011), 845-856.

[32] N. Neisany Samany, M.R. Delavar, N. Chrisman and M.R. Malek, An ontology for spatial relevant objects in a contextaware system: case Study: A tourist guide system, World Academy of Science, Engineering and Technology 63 (2012), $878-884$.

[33] N. Neisany Samany, M.R. Delavar, N. Chrisman and M.R. Malek, Spatial relevancy algorithm for context-aware systems (SRACS) in urban traffic networks using dynamic range neighbor query and directed interval algebra, in press for Journal of Ambient and Smart Environments.

[34] N. Neisany Samany, M.R. Delavar, N. Chrisman and M.R. Malek, Modeling spatio-temporal relevancy in context-aware systems using multi-interval algebra, In: Proceeding of the Joint International Conference and exhibitions on Geomatics2011 and ISPRS Conference on Data Handling and Modeling of Geospatial Information for Management of Resources, 15-16 May 2011, National Cartographic Center of Iran, Tehran.

[35] Q.T. Tran, D. Taniar and M. Safar, Reverse k nearest neighbor and reverse farthest neighbor search on spatial networks, Transactions on Large-Scale Data- and Knowledge-Centered Systems 1 (2009), 353-372.

[36] P.M. Berry, T. Donneau-Golencer, Kh. Duong, M. Gervasio, B. Peintner and N. Yorke-Smith, Evaluating user-adaptive systems: Lessons from experiences with a personalized meeting scheduling assistant, Association for the Advancement of Artificial Intelligence, 2009, (www.aaai.org).

[37] Sh.L. Tsang and S. Clarke, Mining user models for effective adaptation of context-aware applications, Int J Security and its Applications 2(1) (2009), 53-62.

[38] Sh. Wang, D. Liu, J. liu and X. Wang, An algebra for moving objects, In Advances in Spatio-Temporal Analysis. Taylor and Francis Group, London, 2008, pp. 111-122.

[39] T. Olsson, T. Kakkainen, E. Lagerstam and L. Venta- Olkonen, User evaluation of mobile augmented reality scenarios, Journal of Ambient Intelligence and Smart Environments (JAISE) 4 (2012), 29-47.

[40] T. Reichenbacher, The concept of relevance in mobile maps. Location Based Services and Tele-CartographyLecture Notes in Geo-information and Cartography, Section III, 2005, pp. 231-246.

[41] T. Saracevic, Relevance reconsidered, In: Proceeding, The Second Conference on Conceptions of Library and Information Science (CoLIS2), Copenhagen, Denmark, Oct. 14-17, 1996, pp. 201-218. 
[42] W. Pan, Z. Wang and X. Gu, Context-based adaptive personalized web search for improving information retrieval effectiveness. In: Proceeding of IEEE International Conference on Wireless Communications, Networking and Mobile Computing, Shanghai, China, Oct. 8-10, 2007, pp. 5427-5430.

[43] Zh. Pingping, J. Shiguang and Ch. Weihe, A location-based secure spatial audit policy model, International Conference on Computer Science and Software Engineering, IEEE Computer Society, CSSE 4 (2008), 619-622.

[44] zn.wikipedia.org/.

M. Reza Delavar received the BSc. degree in Civil Eng. Surveying from K.N.Toosi Univ. Iran in 1989, M.E. in Civil Eng.Photogrammetry and Remote Sensing from Univ. of Roorkee, India, and a PhD in Geomatic Eng.-GIS from Univ. of New South Wales, Australia in 1997. Since 1998 he has been working in Dept. of Surveying Eng., Eng. Faculty, University of Tehran as an assistant professor. His research interest includes GIS-based artificial intelligence, agent based spatial modeling, spatial data quality, spatio-temporal GIS, land administration, spatial data infrastructure, disaster management and cadastre. He is president of Iranian Society of Surveying and Geomatic Eng. He is also national representative of Iran in UDMS and he is a board member of center of excellence in disaster management in Iran.

Nicholas Chrisman has working in the domain of geographic information for 40 years, developing innovative techniques to analyze and display information about the earth and the people who inhabit it. He has some long term themes in his personal work: addressing data quality, dealing with time and change, and examining institutional and social settings of technology. His specialties are geographic information analysis, geospatial sciences, geomatics, data quality investigations, network of scientists, collaboration, research and innovation networks. For the past 8 years, he managed the GEOIDE Network, a network of researchers and user communities across Canada.

M. Reza Malek is currently an Assistant at the Geodesy and Geomatics Engineering faculty of K.N.Toosi University of Technology. He has more than hundred peer reviewed journal articles, book chapters, and international conference papers. Some of Dr. Malek's research interests include Ubiquitous and Mobile GIS, Spatial analysis and Uncertainty modeling. In May 2005, he received an award for the best researcher in the Planning and Management Organization of Iran from the minister of Research and science of Iran.

Najmeh Neysani Samani received her B.S. degree in Civil Eng.-Surveying engineering from industrial K.N.Toosi Univ, Tehran, Iran in 2004, and M.S. degree in geospatial information system (GIS) engineering from the Tehran University, Tehran, Iran in 2006, being as a GIS researcher in NCC from 2005 to 2008. She is the GIS PhD student from 2007 at Tehran University, Tehran, Iran. Her current research interests are pervasive and ubiquitous computing, spatio-temporal modeling, wayfinding, mobile systems, fuzzy control and approximate reasoning in GIS. 

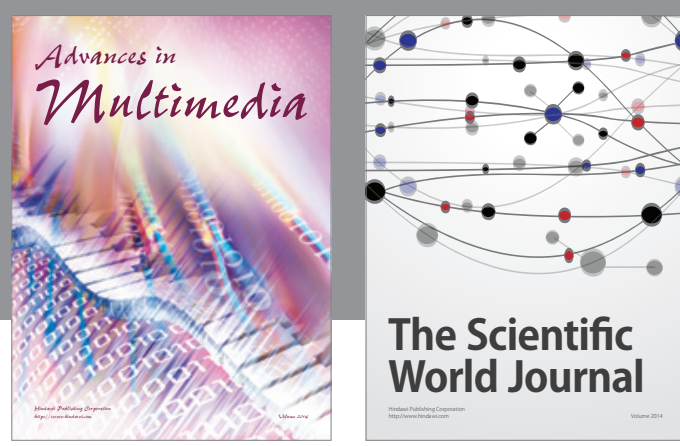

The Scientific World Journal
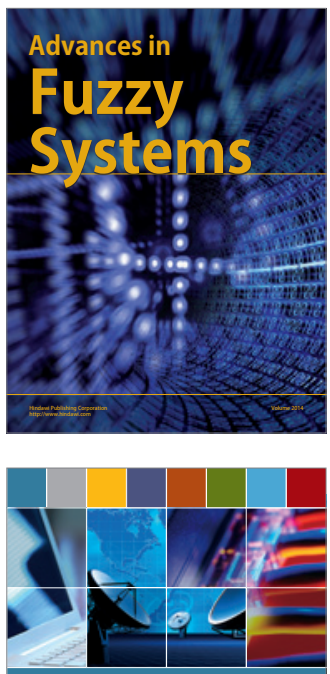

Computer Networks and Communications
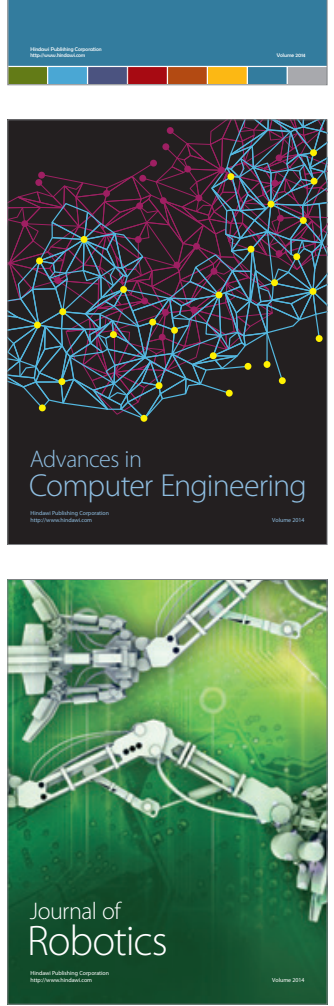
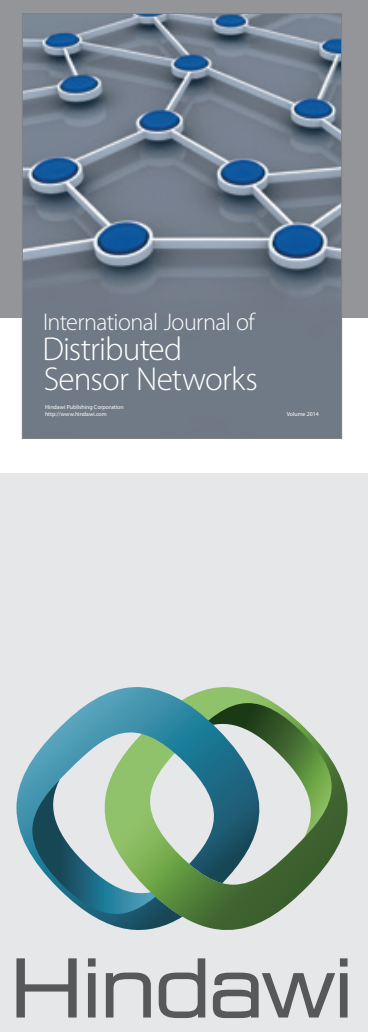

Submit your manuscripts at

http://www.hindawi.com
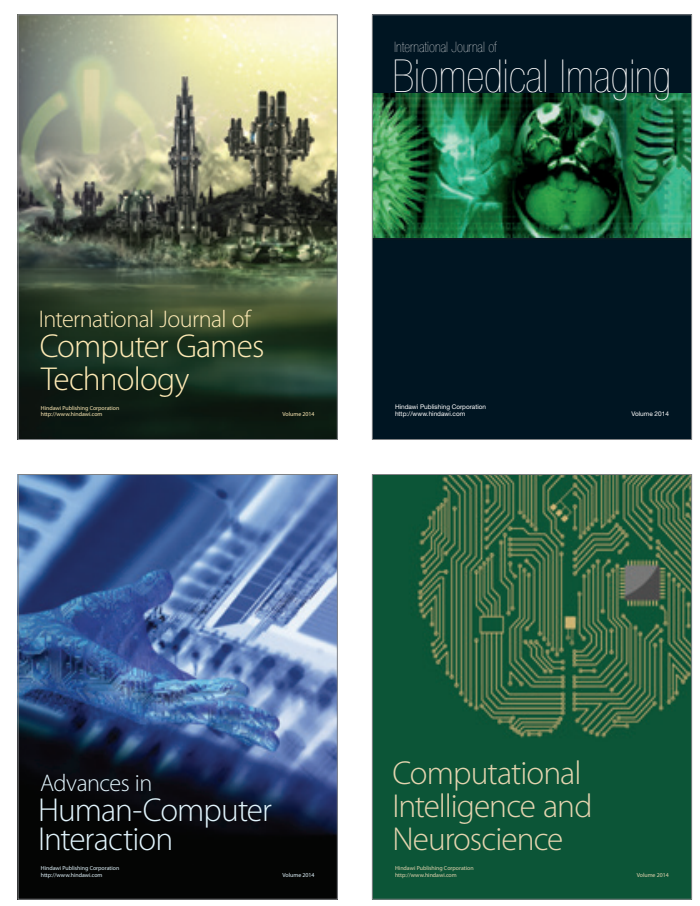
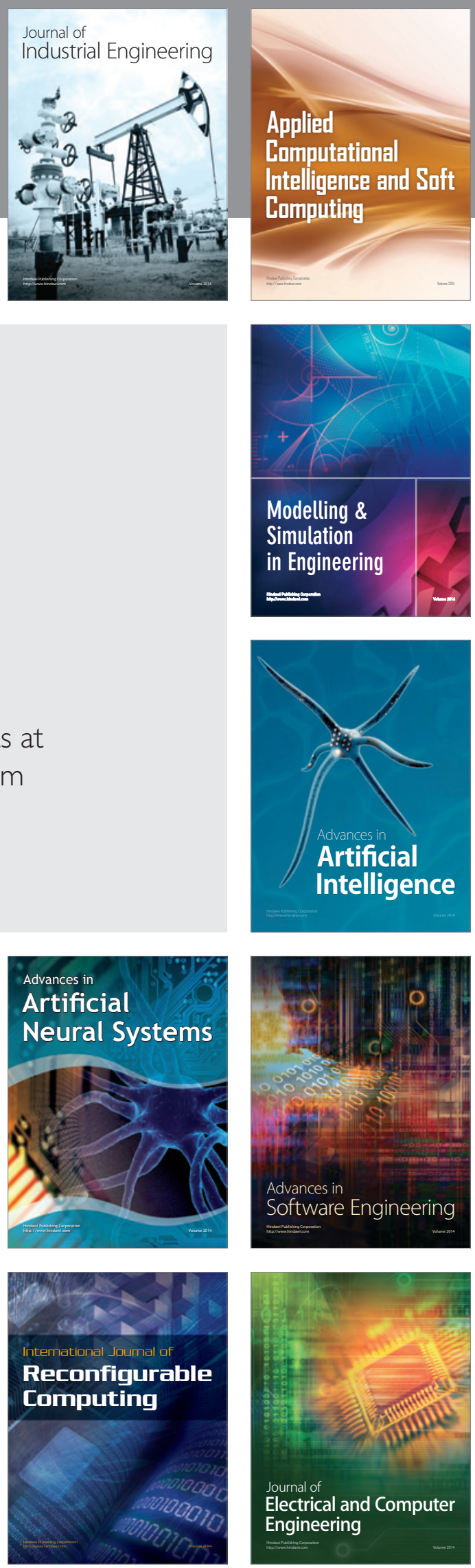